\title{
The local metric dimension of strong product graphs
}

\author{
Gabriel A. Barragán-Ramírez and Juan A. Rodríguez-Velázquez \\ Departament d'Enginyeria Informàtica i Matemàtiques, \\ Universitat Rovira i Virgili, Av. Països Catalans 26, 43007 Tarragona, Spain. \\ gbrbcn@gmail.com, juanalberto.rodriguez@urv.cat
}

September 28, 2018

\begin{abstract}
A vertex $v \in V(G)$ is said to distinguish two vertices $x, y \in V(G)$ of a nontrivial connected graph $G$ if the distance from $v$ to $x$ is different from the distance from $v$ to $y$. A set $S \subset V(G)$ is a local metric generator for $G$ if every two adjacent vertices of $G$ are distinguished by some vertex of $S$. A local metric generator with the minimum cardinality is called a local metric basis for $G$ and its cardinality, the local metric dimension of $G$. It is known that the problem of computing the local metric dimension of a graph is NP-Complete. In this paper we study the problem of finding exact values or bounds for the local metric dimension of strong product of graphs.
\end{abstract}

Keywords: Metric generator; metric dimension; local metric set; local metric dimension, strong product graph.

\section{Introduction}

A metric generator of a metric space $(X, d)$ is a set $S \subset X$ of points in the space with the property that every point of $X$ is uniquely determined by the distances from the elements of $S$. The metric dimension $\operatorname{dim}(X)$ of $(X, d)$ is the smallest integer $t$ such that there is a metric generator of cardinality $t$. A metric generator of cardinality $\operatorname{dim}(X)$ is called a metric basis of $X$.

The concept of metric dimension of a general metric space first appeared in 1953 in 3, but it attracted a little attention, except for the case of graphs. Given a simple and connected graph $G=(V, E)$, defined on the vertex set $V$ and the edge set $E$, we consider the function $d_{G}: V \times V \rightarrow \mathbb{N} \cup\{0\}$, where $d_{G}(x, y)$ is the length of a shortest path between $u$ and $v$ and $\mathbb{N}$ is the set of positive integers. It is readily seen that $\left(V, d_{G}\right)$ is a metric space.

The notion of metric dimension of a graph was introduced by Slater in [27, where the metric generators were called locating sets. Harary and Melter independently introduced the same concept in [14, where metric generators were called resolving sets. Applications of this invariant to the navigation of robots in networks are discussed in [18] and applications to chemistry in [16, 17]. This invariant was studied further in a number of other papers including, for instance [1, 5, 6, 9, 12, 15, 19, 25, 28]. Several variations of metric generators including resolving dominating sets [4], independent resolving sets [7], local metric sets [20], 
strong resolving sets [26], $k$-metric generators [8], simultaneous metric generators [21], etc. have since been introduced and studied.

In this article we are interested in the study of local metric generators, also called local metric sets [20]. A set $S$ of vertices in a connected graph $G$ is a local metric generator for $G$ if every two adjacent vertices of $G$ are distinguished by some vertex of $S$, i.e., for every $u, v \in V(G)$ there exists $s \in S$ such that $d_{G}(u, s) \neq d_{G}(v, s)$. A local metric generator with the minimum cardinality is called a local metric basis for $G$ and its cardinality, the local metric dimension of $\mathrm{G}$, is denoted by $\operatorname{dim}_{l}(G)$. The following main results were obtained in [20].

Theorem 1. 20] Let $G$ be a nontrivial connected graph of order $n$. Then $\operatorname{dim}_{l}(G)=n-1$ if and only if $G$ is complete, and $\operatorname{dim}_{l}(G)=1$ if and only if $G$ is bipartite.

The clique number $\omega(G)$ of a graph $G$ is the order of a largest complete subgraph in $G$.

Theorem 2. 20] Let $G$ be connected graph of order $n$. Then $\operatorname{dim}_{l}(G)=n-2$ if and only if $\omega(G)=n-1$.

The local metric dimension of graphs has been previously studied in $[2,10,11,20,22,23$. In particular, it was shown in [10, 11] that the problem of computing the local metric dimension is NP-Complete. This suggests finding the strong metric dimension for special classes of graphs or obtaining good bounds on this invariant. In this paper we study the problem of finding exact values or sharp bounds for the local metric dimension of strong product graphs.

We begin by giving some basic concepts and notations. For two adjacent vertices $u$ and $v$ of $G=(V, E)$ we use the notation $u \sim v$ and for two isomorphic graphs $G$ and $G^{\prime}$ we use $G \cong G^{\prime}$. For a vertex $v$ of $G, N_{G}(v)$ denotes the set of neighbors that $v$ has in $G$, i.e., $N_{G}(v)=\{u \in V: u \sim v\}$. The set $N_{G}(v)$ is called the open neighborhood of $v$ in $G$ and $N_{G}[v]=N_{G}(v) \cup\{v\}$ is called the closed neighborhood of $v$ in $G$.

We will use the notation $K_{n}, K_{r, s}, C_{n}, N_{n}$ and $P_{n}$ for complete graphs, complete bipartite graphs, cycle graphs, empty graphs and path graphs, respectively.

The strong product of two graphs $G=\left(V_{1}, E_{1}\right)$ and $H=\left(V_{2}, E_{2}\right)$ is the graph $G \otimes H=$ $(V, E)$, such that $V=V_{1} \times V_{2}$ and two vertices $(a, b),(c, d) \in V$ are adjacent in $G \otimes H$ if and only if

$$
\begin{aligned}
& a=c \text { and } b d \in E_{2}, \text { or } \\
& b=d \text { and } a c \in E_{1}, \text { or } \\
& a c \in E_{1} \text { and } b d \in E_{2} .
\end{aligned}
$$

We would point out that the Cartesian product $G \square H$ is a subgraph of $G \otimes H$ and for complete graphs $K_{r} \otimes K_{s}=K_{r s}$.

One of our tools will be a well-known result, which states the relationship between the vertex distances in $G \otimes H$ and the vertex distances in the factor graphs.

Remark 3. [13] Let $G$ and $H$ be two connected graphs. Then

$$
d_{G \otimes H}((a, b),(c, d))=\max \left\{d_{G}(a, c), d_{H}(b, d)\right\} .
$$

For the remainder of the paper, definitions will be introduced whenever a concept is needed. 


\section{General Bounds}

We begin by giving general bounds for the local metric dimension of strong product graphs.

Theorem 4. Let $G$ and $H$ be two connected graphs of order $n_{1} \geq 2$ and $n_{2} \geq 2$, respectively. Then

$$
3 \leq \operatorname{dim}_{l}(G \otimes H) \leq n_{1} \cdot \operatorname{dim}_{l}(H)+n_{2} \cdot \operatorname{dim}_{l}(G)-\operatorname{dim}_{l}(G) \cdot \operatorname{dim}_{l}(H) .
$$

Proof. Let $V_{1}$ and $V_{2}$ be the set of vertices of $G$ and $H$, respectively. We claim that $S=$ $\left(V_{1} \times S_{2}\right) \cup\left(S_{1} \times V_{2}\right)$ is a local metric generator for $G \otimes H$, where $S_{1}$ and $S_{2}$ are local metric basis for $G$ and $H$, respectively.

Let $\left(u_{i}, v_{j}\right),\left(u_{k}, v_{l}\right) \in V_{1} \times V_{2}-S$ be two adjacent vertices of $G \otimes H$. If $i=k$, then $v_{j}$ and $v_{l}$ are adjacent in $H$ and there exists $b \in S_{2}$ such that $d_{G \otimes H}\left(\left(u_{i}, b\right),\left(u_{i}, v_{j}\right)\right)=$ $d_{H}\left(b, v_{j}\right) \neq d_{H}\left(b, v_{l}\right)=d_{G \otimes H}\left(\left(u_{i}, b\right),\left(u_{k}, v_{l}\right)\right)$. So, $\left(u_{i}, v_{j}\right)$ and $\left(u_{k}, v_{l}\right)$ are distinguished by $\left(u_{i}, b\right) \in\left(V_{1} \times S_{2}\right) \subset S$. Analogously, if $j=l$, then $u_{i}$ and $u_{k}$ are adjacent in $G$ and there exists $a \in S_{1}$ such that $d_{G}\left(a, u_{i}\right) \neq d_{G}\left(a, u_{k}\right)$ and, as above, $\left(u_{i}, v_{j}\right)$ and $\left(u_{k}, v_{l}\right)$ are distinguished by $\left(a, v_{j}\right) \in\left(S_{1} \times V_{2}\right) \subset S$. Finally, if $u_{i} u_{k} \in E_{1}$ and $v_{j} v_{l} \in E_{2}$, then for any $a \in S_{1}$ such that $d_{G}\left(a, u_{i}\right) \neq d_{G}\left(a, u_{k}\right)$ we have

$$
d_{G \bowtie H}\left(\left(u_{i}, v_{j}\right),\left(a, v_{j}\right)\right)=d_{G}\left(u_{i}, a\right) \neq d_{G}\left(u_{k}, a\right)=\max \left\{d_{G}\left(u_{k}, a\right), 1\right\}=d_{G \bowtie H}\left(\left(a, v_{j}\right),\left(u_{k}, v_{l}\right)\right) .
$$

Thus, $\left(u_{i}, v_{j}\right)$ and $\left(u_{k}, v_{l}\right)$ are distinguished by $\left(a, v_{j}\right) \in S_{1} \times V_{2} \subset S$. Then we conclude that $S$ is a local metric generator for $G \otimes H$ and, as a consequence, $\operatorname{dim}_{l}(G \otimes H) \leq|S|=$ $n_{1} \cdot \operatorname{dim}_{l}(H)+n_{2} \cdot \operatorname{dim}_{l}(G)-\operatorname{dim}_{l}(G) \cdot \operatorname{dim}_{l}(H)$.

To prove the lower bound, let $B$ be a local metric basis of $G \otimes H$. Given $\left(u_{1}, v_{1}\right) \in B$, chose $u^{*} \in N_{G}\left(u_{1}\right), v^{*} \in N_{H}\left(v_{1}\right)$ and define

$$
W=\left\{\left(u^{*}, v_{1}\right),\left(u_{1}, v^{*}\right),\left(u^{*}, v^{*}\right)\right\} .
$$

Since $\left(u_{1}, v_{1}\right)$ is not able to distinguish any pair of adjacent vertices in $W$, there exists $\left(u_{2}, v_{2}\right) \in B-\left\{\left(u_{1}, v_{1}\right)\right\}$. Let

$$
q=\min _{(a, b) \in W}\left\{d_{G \otimes H}\left(\left(u_{2}, v_{2}\right),(a, b)\right)\right\} .
$$

Now, as $d_{G \otimes H}\left((a, b),\left(u_{2}, v_{2}\right)\right) \in\{q, q+1\}$ for every $(a, b) \in W$, by Dirichlet's box principle, there are two vertices $\left(x_{1}, y_{1}\right),\left(x_{2}, y_{2}\right) \in W$ such that

$$
d_{G \otimes H}\left(\left(u_{2}, v_{2}\right),\left(x_{1}, y_{1}\right)\right)=d_{G \otimes H}\left(\left(u_{2}, v_{2}\right),\left(x_{2}, y_{2}\right)\right) .
$$

Hence, $B-\left\{\left(u_{1}, v_{1}\right),\left(u_{2}, v_{2}\right)\right\} \neq \emptyset$, and the result follows.

Since $K_{n_{1}} \otimes K_{n_{2}} \cong K_{n_{1} \cdot n_{2}}$ and for any complete graph $K_{n}, \operatorname{dim}_{l}\left(K_{n}\right)=n-1$, we deduce $\operatorname{dim}_{l}\left(K_{n_{1}} \otimes K_{n_{2}}\right)=n_{1} \cdot n_{2}-1=n_{1} \cdot \operatorname{dim}_{l}\left(K_{n_{2}}\right)+n_{2} \cdot \operatorname{dim}_{l}\left(K_{n_{1}}\right)-\operatorname{dim}_{l}\left(K_{n_{1}}\right) \cdot \operatorname{dim}_{l}\left(K_{n_{2}}\right)$.

Therefore, the upper bound is tight. Examples of non-complete graphs, where the upper bound is attained, can be derived from Theorem 10 .

In order to show that the lower bound is tight, consider two paths $P_{t}$ and $P_{t^{\prime}}$, where $t^{\prime} \leq t \leq 2 t^{\prime}-1, V\left(P_{t}\right)=\left\{u_{1}, u_{2}, \ldots, u_{t}\right\}$ and $u_{i} \sim u_{i+1}$, for every $i \in\{1, \ldots, t-1\}$. Also, take $v_{1}, v_{t^{\prime}} \in V\left(P_{t^{\prime}}\right)$ such that $d_{P_{t^{\prime}}}\left(v_{1}, v_{t^{\prime}}\right)=t^{\prime}-1$. It is not difficult to check that $\left\{\left(u_{1}, v_{1}\right),\left(u_{t^{\prime}}, v_{t^{\prime}}\right),\left(u_{t}, v_{1}\right)\right\}$ is a local metric generator for $P_{t} \otimes P_{t^{\prime}}$, so that Theorem 4 leads to $\operatorname{dim}_{l}\left(P_{t} \otimes P_{t^{\prime}}\right)=3$. 


\section{The Particular Case of Adjacency $k$-Resolved Graphs}

Now we will give some results involving the diameter or the radius of $G$. The eccentricity $\epsilon(v)$ of a vertex $v$ in a connected graph $G$ is the maximum distance between $v$ and any other vertex $u$ of $H$. So, the diameter of $G$ is defined as

$$
D(G)=\max _{v \in V(G)}\{\epsilon(v)\}
$$

while the radius is defined as

$$
r(G)=\min _{v \in V(G)}\{\epsilon(v)\} .
$$

Given two vertices $x$ and $y$ in a connected graph $G=(V, E)$, the interval $I[x, y]$ between $x$ and $y$ is defined as the collection of all vertices which lie on some shortest $x-y$ path. Given a nonnegative integer $k$, we say that $G$ is adjacency $k$-resolved if for every two adjacent vertices $x, y \in V$, there exists $w \in V$ such that

$$
\begin{aligned}
& d_{G}(y, w) \geq k \text { and } x \in I[y, w], \text { or } \\
& d_{G}(x, w) \geq k \text { and } y \in I[x, w] .
\end{aligned}
$$

For instance, the path and the cycle graphs of order $n(n \geq 2)$ are adjacency $\left\lceil\frac{n}{2}\right\rceil$ resolved, the two-dimensional grid graphs $P_{r} \square P_{t}$ are adjacency $\left(\left\lceil\frac{r}{2}\right\rceil+\left\lceil\frac{t}{2}\right\rceil\right)$-resolved, and the hypercube graphs $Q_{k}$ are adjacency $k$-resolved.

Theorem 5. Let $H$ be an adjacency $k$-resolved graph of order $n_{2}$ and let $G$ be a non-trivial graph of diameter $D(G)<k$. Then $\operatorname{dim}_{l}(G \otimes H) \leq n_{2} \cdot \operatorname{dim}_{l}(G)$.

Proof. Let $V_{1}=\left\{u_{1}, u_{2}, \ldots, u_{n_{1}}\right\}$ and $V_{2}=\left\{v_{1}, v_{2}, \ldots, v_{n_{2}}\right\}$ be the set of vertices of $G$ and $H$, respectively. Let $S_{1}$ be a local metric generator for $G$. We will show that $S=S_{1} \times V_{2}$ is a local metric generator for $G \otimes H$. Let $\left(u_{i}, v_{j}\right),\left(u_{r}, v_{l}\right)$ be two adjacent vertices of $G \otimes H$. We differentiate the following two cases.

Case 1. $j=l$. Since $u_{i} \sim u_{r}$ and $S_{1}$ is a local metric generator for $G$, there exists $u \in S_{1}$ such that $d_{G}\left(u_{i}, u\right) \neq d_{G}\left(u_{r}, u\right)$. Hence,

$$
d_{G \otimes H}\left(\left(u_{i}, v_{j}\right),\left(u, v_{j}\right)\right)=d_{G}\left(u_{i}, u\right) \neq d_{G}\left(u_{r}, u\right)=d_{G \otimes H}\left(\left(u_{r}, v_{j}\right),\left(u, v_{j}\right)\right) .
$$

Case 2. $v_{j} \sim v_{l}$. Since $H$ is adjacency $k$-resolved, there exists $v \in V_{2}$ such that $\left(d_{H}\left(v, v_{l}\right) \geq k\right.$ and $\left.v_{j} \in I\left[v, v_{l}\right]\right)$ or $\left(d_{H}\left(v, v_{j}\right) \geq k\right.$ and $\left.v_{l} \in I\left[v, v_{j}\right]\right)$. Say $d_{H}\left(v, v_{l}\right) \geq k$ and $v_{j} \in I\left[v, v_{l}\right]$. In such a case, as $D(G)<k$, for every $u \in S_{1}$ we have

$$
\begin{aligned}
d_{G \otimes H}\left(\left(u_{i}, v_{j}\right),(u, v)\right) & =\max \left\{d_{G}\left(u_{i}, u\right), d_{H}\left(v_{j}, v\right)\right\} \\
& <d_{H}\left(v, v_{l}\right) \\
& =\max \left\{d_{G}\left(u, u_{r}\right), d_{H}\left(v, v_{l}\right)\right\} \\
& =d_{G \otimes H}\left(\left(u_{r}, v_{l}\right),(u, v)\right) .
\end{aligned}
$$

Therefore, $S$ is a local metric generator for $G \otimes H$.

Lemma 6. Let $H$ be a connected bipartite graph of order greater than or equal to three. Then $H$ is adjacency $k$-resolved for any $k \in\{2, . ., r(H)\}$. 
Proof. Let $x, y, w \in V(H)$ such that $x \sim y$ and $d_{H}(x, w)=k$, for some $k \in\{2, . ., r(H)\}$. Since $H$ does not have cycles of odd length, $d_{H}(w, y) \neq k$. Thus, either $d_{H}(w, y)=d_{H}(w, x)+$ $d_{H}(x, y)=k+1$ or $d_{H}(w, x)=d_{H}(w, y)+d_{H}(y, x)=k$. Therefore, the result follows.

Now we derive a consequences of combining Theorem 5 and Lemma 6 ,

Theorem 7. Let $G$ and $H$ be two connected non-trivial graphs. If $H$ is bipartite and $D(G)<$ $r(H)$, then $\operatorname{dim}_{l}(G \otimes H) \leq|V(H)| \operatorname{dim}_{l}(G)$.

As we will show in Theorem 14, the above inequality is tight.

\section{The Role of True Twin Equivalence Classes}

Two vertices $u$ and $v$ of a graph $G$ are true twins if $N_{G}[u]=N_{G}[v]$. Note that if two vertices $u$ and $v$ of a graph $G$ are true twins, then $d_{G}(x, u)=d_{G}(x, v)$, for every $x \in V(G)-\{u, v\}$. We define the true twin equivalence relation $\mathcal{R}$ on $V(G)$ as follows:

$$
x \mathcal{R} y \longleftrightarrow N_{G}[x]=N_{G}[y] .
$$

If the true twin equivalence classes are $U_{1}, U_{2}, \ldots, U_{t}$, then every local metric generator of $G$ must contain at least $\left|U_{i}\right|-1$ vertices from $U_{i}$, for each $i \in\{1, \ldots, t\}$. Thus the following result presented in [20] holds.

Theorem 8. [20] If $G$ is a nontrivial connected graph of order $n$ having t true twin equivalence classes, then $\operatorname{dim}_{l}(G) \geq n-t$.

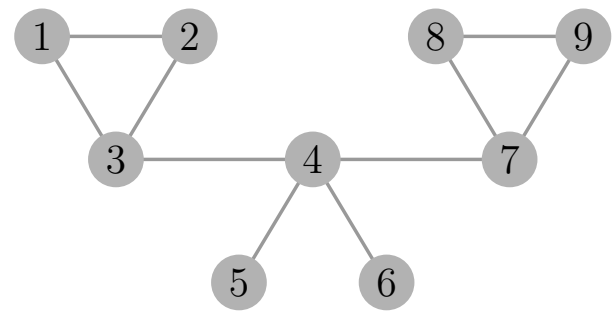

Figure 1: This graph has $t=7$ true twin equivalence classes; two of them are $\{1,2\}$ and $\{8,9\}$ and the remain classes are singleton sets. A local metric basis is $\{1,9\}$ while a metric basis is $\{1,5,9\}$. Thus, $\operatorname{dim}_{l}(G)=n-t=2<3=\operatorname{dim}(G)$.

Note that the complete graph has only one true twin equivalence class and in any triangle-free graph all the true twin equivalence classes are singleton. As an example of noncomplete graph $G$ of order $n$ having $t$ true twin equivalence classes, where $\operatorname{dim}_{l}(G)=n-t$, we take $G=K_{1}+\left(\bigcup_{i=1}^{l} K_{r_{i}}\right), r_{i} \geq 2, l \geq 2$. In this case $G$ has $t=l+1$ true twin equivalence classes, $n=1+\sum_{i=1}^{l} r_{i}$ and $\operatorname{dim}_{l}(G)=\sum_{i=1}^{l}\left(r_{i}-1\right)=n-t$. Figure 1 shows another example of graph where the bound given in Theorem 8 is reached.

Lemma 9. Let $G$ and $H$ be two non-trivial connected graphs of order $n_{1}$ and $n_{2}$, having $t_{1}$ and $t_{2}$ true twin equivalent classes, respectively. Then the vertex set of $G \otimes H$ is partitioned into $t_{1} t_{2}$ true twin equivalent classes. 
Proof. First of all, we would point out that for any $a \in V(G)$ and $b \in V(H)$ it holds

$$
N_{G \otimes H}[(a, b)]=\left\{(x, y): x \in N_{G}[a], y \in N_{H}[b]\right\}=N_{G}[a] \times N_{H}[b] .
$$

Now, since the result immediately holds for complete graphs, we assume that $G \nsubseteq K_{n_{1}}$ or $H \nsubseteq K_{n_{2}}$. Let $U_{1}, U_{2}, \ldots, U_{t_{1}}$ and $U_{1}^{\prime}, U_{2}^{\prime}, \ldots, U_{t_{2}}^{\prime}$ be the true twin equivalence classes of $G$ and $H$, respectively. Since each $U_{i}$ (and $U_{j}^{\prime}$ ) induces a clique and its vertices have identical closed neighbourhoods, for every $a, c \in U_{i}$ and $b, d \in U_{j}^{\prime}$,

$$
N_{G \otimes H}[(a, b)]=N_{G}[a] \times N_{H}[b]=N_{G}[c] \times N_{H}[d]=N_{G \otimes H}[(c, d)] .
$$

Hence, $V(G) \times V(H)$ is partitioned as $V(G) \times V(H)=\bigcup_{j=1}^{t_{2}}\left(\bigcup_{i=1}^{t_{1}} U_{i} \times U_{j}^{\prime}\right)$, where $U_{i} \times U_{j}^{\prime}$ induces a clique in $G \otimes H$ and its vertices have identical closed neighbourhoods. Moreover, for any $(a, b) \in U_{i} \times U_{j}^{\prime}$ and $(c, d) \in U_{k} \times U_{l}^{\prime}$, where $i \neq k$ or $j \neq l$, we have

$$
N_{G \otimes H}[(a, b)]=N_{G}[a] \times N_{H}[b] \neq N_{G}[c] \times N_{H}[d]=N_{G \otimes H}[(c, d)] .
$$

Therefore, the true twin equivalence classes of $G \otimes H$ are of the form $U_{i} \times U_{j}^{\prime}$, where $i \in$ $\left\{1, . ., t_{1}\right\}$ and $j \in\left\{1, . ., t_{2}\right\}$.

We would point out that the above result was indirectly obtained in [24], proof of Theorem 2.3.

Theorem 8 and Lemma 9 directly lead to the next result.

Theorem 10. Let $G$ and $H$ be two non-trivial connected graphs of order $n_{1}$ and $n_{2}$, having $t_{1}$ and $t_{2}$ true twin equivalence classes, respectively. Then

$$
\operatorname{dim}_{l}(G \otimes H) \geq n_{1} n_{2}-t_{1} t_{2}
$$

By Theorems 1, 4 and 10 we deduce the following result.

Theorem 11. Let $G$ and $H$ be two non-trivial connected graphs of order $n_{1}$ and $n_{2}$, having $t_{1}$ and $t_{2}$ true twin equivalence classes, respectively. Then the following assertions hold:

(i) If $\operatorname{dim}_{l}(G)=n_{1}-t_{1}$ and $\operatorname{dim}_{l}(H)=n_{2}-t_{2}$, then $\operatorname{dim}_{l}(G \otimes H)=n_{1} n_{2}-t_{1} t_{2}$.

(ii) If $\operatorname{dim}_{l}(G)=n_{1}-t_{1}$ and $H$ is bipartite, then $n_{2}\left(n_{1}-t_{1}\right) \leq \operatorname{dim}_{l}(G \otimes H) \leq n_{2}\left(n_{1}-t_{1}\right)+t_{1}$.

Since any complete graph $K_{n}$ has only one true twin equivalence class, Theorem 11leads to the next result.

Corollary 12. Let $H$ be a connected graph of order $n^{\prime} \geq 2$ having $t$ true twin equivalent classes. Then for any integer $n \geq 2$,

$$
\operatorname{dim}_{l}\left(K_{n} \otimes H\right)=n n^{\prime}-t .
$$

In particular, if $H$ does not have true twin vertices, then

$$
\operatorname{dim}_{l}\left(K_{n} \otimes H\right)=n^{\prime}(n-1)
$$

Note that if $H$ is an adjacency $k$-resolved graph, for $k \geq 2$, then $H$ does not have true twin vertices. Therefore, Theorems 10 and 5 lead to the following result. 
Theorem 13. Let $H$ be an adjacency $k$-resolved graph of order $n_{2}$ and let $G$ be a non-trivial connected graph of order $n_{1}$, having $t_{1}$ true twin equivalence classes and diameter $D(G)<k$. If $\operatorname{dim}_{l}(G)=n_{1}-t_{1}$, then $\operatorname{dim}_{l}(G \otimes H)=n_{2}\left(n_{1}-t_{1}\right)$.

Our next result can be deduced from Corollary 6 and Theorem 13 or from Theorems 10 and 7 .

Theorem 14. Let $H$ be connected bipartite graph of order $n_{2}$ and let $G$ be a non-trivial connected graph of order $n_{1}$, having $t_{1}$ true twin equivalence classes. If $\operatorname{dim}_{l}(G)=n_{1}-t_{1}$ and $D(G)<r(H)$, then $\operatorname{dim}_{l}(G \otimes H)=n_{2}\left(n_{1}-t_{1}\right)$.

\section{The Particular Case of $P_{t} \otimes G$}

In this section we assume that $t$ is an integer greater than or equal to two and $V\left(P_{t}\right)=$ $\left\{u_{1}, u_{2}, \ldots, u_{t}\right\}$, where $u_{i} \sim u_{i+1}$, for every $i \in\{1, \ldots, t-1\}$. In the proof of the next lemma we will use the notation $\mathcal{B}_{r}(x)$ for the closed ball of center $x \in V(G)$ and radius $r \geq 0$, i.e.,

$$
\mathcal{B}_{r}(x) .=\left\{y \in V(G): d_{G}(x, y) \leq r\right\} .
$$

Lemma 15. Let $G$ be a connected graph and let $t \geq 1$ be an integer. Let $u_{i_{1}}, u_{i_{2}}, \ldots, u_{i_{b}}$ be the first components of the elements in a local metric basis of $P_{t} \otimes G$, where $i_{1} \leq i_{2} \leq \cdots \leq i_{b}$. Then the following assertions hold.

(i) $i_{2} \leq D(G)+1$ and $i_{b-1} \geq t-D(G)$.

(ii) For any $l \in\{1, \ldots, b-2\}, i_{l+2} \leq 2 D(G)+i_{l}$.

(iii) $i_{3} \leq 2 D(G)+1$.

Proof. Let $B$ be a local metric basis of $P_{t} \otimes G$ and let $u_{i_{1}}, u_{i_{2}}, \ldots, u_{i_{b}}$ be the first components of the elements in $B$, where $i_{1} \leq i_{2} \leq \cdots \leq i_{b}$. First of all, notice that $|B|=b$ and, by Theorem $4, b \geq 3$.

We first proceed to prove (i). Suppose, for the contrary, that $i_{2}>D(G)+1$. Let $y, z \in V(G)$ such that $\left(u_{i_{1}}, y\right) \in B$ and $z \in N_{G}(y)$. If $i_{1} \neq 1$, then no vertex in $B$ is able to distinguish $\left(u_{1}, y\right)$ and $\left(u_{1}, z\right)$. Now, if $i_{1}=1$, then no vertex in $B$ is able to distinguish $\left(u_{2}, y\right)$ and $\left(u_{2}, z\right)$. So, in both cases we get a contradiction. The proof of $i_{b-1} \geq t-D(G)$ is deduced by symmetry. Hence, (i) follows.

To prove (ii) we proceed by contradiction. Suppose that $i_{l+2}>2 D(G)+i_{l}$ for some $l \in$ $\{1, \ldots, b-2\}$. In such a case we have that $i_{l+1}>D(G)+i_{l}$ or $i_{l+2}>D(G)+i_{l+1}$. We suppose that $i_{l+1}>D(G)+i_{l}$, being the second case analogous. We now take $y, z \in V(G)$ such that $\left(u_{i_{l+1}}, y\right) \in B$ and $z \in N_{G}(y)$. Notice that $\left(u_{i_{l}+D(G)}, y\right)$ and $\left(u_{i_{l}+D(G)}, z\right)$ are adjacent.We differentiate the following cases for $\left(u_{i_{k}}, w\right) \in B$. If $k \leq l$, then $i_{l}+D(G)-i_{k} \geq D(G)$ and so

$$
d_{P_{t} \bowtie G}\left(\left(u_{i_{k}}, w\right),\left(u_{i_{l}+D(G)}, y\right)\right)=i_{l}+D(G)-i_{k}=d_{P_{t} \bowtie G}\left(\left(u_{i_{k}}, w\right),\left(u_{i_{l}+D(G)}, z\right)\right) .
$$

If $k=l+1$ and $i_{l+1} \neq i_{l+2}$, then $w=y$ and since $i_{l+1}>D(G)+i_{l}$, we have

$$
d_{P_{t} \bowtie G}\left(\left(u_{i_{k}}, w\right),\left(u_{i_{l}+D(G)}, y\right)\right)=i_{k}-i_{l}-D(G)=d_{P_{t} \bowtie G}\left(\left(u_{i_{k}}, w\right),\left(u_{i_{l}+D(G)}, z\right)\right) .
$$

If $k=l+1$ and $i_{l+1}=i_{l+2}$, then from the assumption $i_{l+2}>2 D(G)+i_{l}$ we have that $i_{k}-i_{l}-D(G)>D(G)$ and so

$$
d_{P_{t} \bowtie G}\left(\left(u_{i_{k}}, w\right),\left(u_{i_{l}+D(G)}, y\right)\right)=i_{k}-i_{l}-D(G)=d_{P_{t} \bowtie G}\left(\left(u_{i_{k}}, w\right),\left(u_{i_{l}+D(G)}, z\right)\right) .
$$


If $k \geq l+2$, then the assumption $i_{l+2}>2 D(G)+i_{l}$ leads to $i_{k}-i_{l}-D(G)>D(G)$ and so

$$
d_{P_{t} \bowtie G}\left(\left(u_{i_{k}}, w\right),\left(u_{i_{l}+D(G)}, y\right)\right)=i_{k}-i_{l}-D(G)=d_{P_{t} \bowtie G}\left(\left(u_{i_{k}}, w\right),\left(u_{i_{l}+D(G)}, z\right)\right) .
$$

Hence, no vertex in $B$ is able to distinguish $\left(u_{i_{l}+D(G)}, y\right)$ from $\left(u_{i_{l}+D(G)}, z\right)$, which is a contradiction. Therefore, the proof of (ii) is complete.

Finally, we proceed to prove (iii). If $i_{1}=1$, then by (ii) we obtain $i_{3} \leq 2 D(G)+1$. Hence, we assume that $i_{1}>1$. For contradiction purposes, suppose that $i_{3}>2 D(G)+1$. We differentiate two cases for $\left(u_{i_{1}}, v_{1}\right),\left(u_{i_{2}}, v_{2}\right) \in B$.

Case 1: $i_{1}+i_{2}-2>d_{G}\left(v_{1}, v_{2}\right)$. In this case $\left|\mathcal{B}_{i_{1}-1}\left(v_{1}\right) \cap \mathcal{B}_{i_{2}-1}\left(v_{2}\right)\right| \geq 2$ and so we take $\alpha, \beta \in \mathcal{B}_{i_{1}-1}\left(v_{1}\right) \cap \mathcal{B}_{i_{2}-1}\left(v_{2}\right)$ such that $\alpha \sim \beta$. For the pair of adjacent vertices $\left(u_{1}, \alpha\right),\left(u_{1}, \beta\right)$ we have

$$
d_{P_{t} \bowtie G}\left(\left(u_{i_{1}}, v_{1}\right),\left(u_{1}, \alpha\right)\right)=i_{1}-1=d_{P_{t} \bowtie G}\left(\left(u_{i_{1}}, v_{1}\right),\left(u_{1}, \beta\right)\right)
$$

and

$$
d_{P_{t} \bowtie G}\left(\left(u_{i_{2}}, v_{2}\right),\left(u_{1}, \alpha\right)\right)=i_{2}-1=d_{P_{t} \bowtie G}\left(\left(u_{i_{2}}, v_{2}\right),\left(u_{1}, \beta\right)\right) .
$$

So, neither $\left(u_{i_{1}}, v_{1}\right)$ nor $\left(u_{i_{2}}, v_{2}\right)$ distinguishes $\left(u_{1}, \alpha\right)$ from $\left(u_{1}, \beta\right)$. Furthermore, for $i_{r} \geq$ $i_{3}>2 D(G)+1$ and $\left(u_{i_{r}}, v_{r}\right) \in B$ we have

$$
d_{P_{t} \bowtie G}\left(\left(u_{i_{r}}, v_{r}\right),\left(u_{1}, \alpha\right)\right)=i_{r}-1=d_{P_{t} \bowtie G}\left(\left(u_{i_{r}}, v_{r}\right),\left(u_{1}, \beta\right)\right) .
$$

Therefore, no vertex $\left(u_{i_{r}}, v_{r}\right) \in B$ distinguishes $\left(u_{1}, \alpha\right)$ from $\left(u_{1}, \beta\right)$, which is a contradiction. Case 2: $i_{1}+i_{2}-2 \leq d\left(v_{1}, v_{2}\right)$. In this case we have

$$
\left(D(G)+2-i_{1}\right)+\left(D(G)+2-i_{2}\right)=2 D(G)+2-\left(i_{1}+i_{2}-2\right) \geq 2 D(G)+2-d\left(v_{1}, v_{2}\right) \geq D(G)+2 .
$$

Hence, there exist $\alpha, \beta \in \mathcal{B}_{D(G)+2-i_{1}}\left(v_{1}\right) \cap \mathcal{B}_{D(G)+2-i_{2}}\left(v_{2}\right)$ such that $\alpha \sim \beta$. For the pair of adjacent vertices $\left(u_{D(G)+2}, \alpha\right),\left(u_{D(G)+2}, \beta\right)$ we have

$$
d_{P_{t} \bowtie G}\left(\left(u_{i_{1}}, v_{1}\right),\left(u_{D(G)+2}, \alpha\right)\right)=D(G)+2-i_{1}=d_{P_{t} \bowtie G}\left(\left(u_{i_{1}}, v_{1}\right),\left(u_{D(G)+2}, \beta\right)\right)
$$

and

$$
d_{P_{t} \bowtie G}\left(\left(u_{i_{2}}, v_{2}\right),\left(u_{D(G)+2}, \alpha\right)\right)=D(G)+2-i_{2}=d_{P_{t} \bowtie G}\left(\left(u_{i_{2}}, v_{2}\right),\left(u_{D(G)+2}, \beta\right)\right)
$$

So, neither $\left(u_{i_{1}}, v_{1}\right)$ nor $\left(u_{i_{2}}, v_{2}\right)$ distinguishes $\left(u_{D(G)+2}, \alpha\right)$ from $\left(u_{D(G)+2}, \beta\right)$. For $i_{r} \geq i_{3}>$ $2 D(G)+1$ and $\left(u_{i_{r}}, v_{r}\right) \in B$ we have

$$
d_{P_{t} \bowtie G}\left(\left(u_{i_{r}}, v_{r}\right),\left(u_{D(G)+2}, \alpha\right)\right)=i_{r}-(D(G)+2)=d_{P_{t} \bowtie G}\left(\left(u_{i_{r}}, v_{r}\right),\left(u_{D(G)+2}, \beta\right)\right) .
$$

Thus, no vertex $\left(u_{i_{r}}, v_{r}\right) \in B$ distinguishes $\left(u_{D(G)+2}, \alpha\right)$ from $\left(u_{D(G)+2}, \beta\right)$, which is a contradiction.

Theorem 16. For any connected $G$ and any integer $t \geq 2 D(G)+1$,

$$
\operatorname{dim}_{l}\left(P_{t} \otimes G\right) \geq\left\lceil\frac{t-1}{D(G)}\right\rceil+1 .
$$


Proof. Let $B$ be a local metric basis of $P_{t} \otimes G$ and let $u_{i_{1}}, u_{i_{2}}, \ldots, u_{i_{b}}$ be the first components of the elements in $B$, where $i_{1} \leq i_{2} \leq \cdots \leq i_{b}$. We differentiate two cases.

Case 1. $b$ odd. In this case $b-1$ is even and by Lemma 15 (i) and (ii) we have

$$
i_{2} \leq D(G)+1, i_{4} \leq 3 D(G)+1, \ldots, i_{b-1} \leq(b-2) D(G)+1
$$

Case 2. $b$ even. In this case $b-1$ is odd and by Lemma 15 (iii) and (ii) we have

$$
i_{3} \leq 2 D(G)+1, i_{5} \leq 4 D(G)+1, \ldots, i_{b-1} \leq(b-2) D(G)+1
$$

According to the two cases above and Lemma 15 (i) we have

$$
t-D(G) \leq i_{b-1} \leq(b-2) D(G)+1 .
$$

Therefore, $b \geq \frac{t-1}{D(G)}+1$.

From now on we say that a set $W \subset V(G \otimes H)$ resolves the set $X \subseteq V(G \otimes H)$ if every pair of adjacent vertices in $X$ is distinguished by some element in $W$.

Lemma 17. Let $G$ and $H$ be two connected nontrivial graphs such that $H$ is bipartite. Let $u_{1}, u_{2}, u_{3} \in V(G)$ and $v_{1}, v_{2} \in V(H)$ such that $u_{2} \in I_{G}\left[u_{1}, u_{3}\right], d_{G}\left(u_{1}, u_{2}\right) \leq d_{H}\left(v_{1}, v_{2}\right)=$ $D(H)$ and $d_{G}\left(u_{2}, u_{3}\right) \geq D(H)$. Then, for any shortest path $P$ from $u_{1}$ to $u_{2}$, the set $B=$ $\left\{\left(u_{1}, v_{1}\right),\left(u_{2}, v_{2}\right),\left(u_{3}, v_{1}\right)\right\}$ resolves $V(P) \times V(H)$.

Proof. Let $P$ be a shortest path form $u_{1}$ to $u_{2}$ and let $\left(u_{i}, v_{j}\right),\left(u_{k}, v_{l}\right) \in V(G \otimes H)$ be two adjacent vertices such that $u_{i}, u_{k} \in V(P)$. Without lost of generality, we assume that $d_{G}\left(u_{i}, u_{1}\right) \leq$ $d_{G}\left(u_{k}, u_{1}\right)$. Notice that from this assumption we have that $d_{G}\left(u_{i}, u_{3}\right) \geq d_{G}\left(u_{k}, u_{3}\right)$. We differentiate the following two cases:

Case 1: $u_{i} \sim u_{k}$. As $d_{G}\left(u_{2}, u_{3}\right) \geq D(H)$ and $u_{i}, u_{k} \in V(P)$, we have $D(H) \leq d_{G}\left(u_{3}, u_{k}\right)<$ $d_{G}\left(u_{3}, u_{i}\right)$ and so $d_{G \otimes H}\left(\left(u_{3}, v_{1}\right),\left(u_{i}, v_{j}\right)\right)=d_{G}\left(u_{3}, u_{i}\right)>d_{G}\left(u_{3}, u_{k}\right)=d_{G \otimes H}\left(\left(u_{3}, v_{1}\right),\left(u_{k}, v_{l}\right)\right)$.

Case 2: $i=k$. In this case $v_{j} \sim v_{l}$ and, as $H$ is a bipartite graph, $d_{H}\left(v_{1}, v_{j}\right) \neq d_{H}\left(v_{1}, v_{l}\right)$ and $d_{H}\left(v_{2}, v_{j}\right) \neq d_{H}\left(v_{2}, v_{l}\right)$. We assume, without lost of generality, that $d_{H}\left(v_{1}, v_{j}\right)<d_{H}\left(v_{1}, v_{l}\right)$. Notice that

$$
d_{H}\left(v_{1}, v_{j}\right)+d_{H}\left(v_{j}, v_{2}\right) \geq d_{H}\left(v_{1}, v_{2}\right)=D(H) \geq d_{G}\left(u_{1}, u_{2}\right)=d_{G}\left(u_{1}, u_{i}\right)+d_{G}\left(u_{i}, u_{2}\right) .
$$

Hence, $d_{H}\left(v_{1}, v_{j}\right) \geq d_{G}\left(u_{1}, u_{i}\right)$ or $d_{H}\left(v_{j}, v_{2}\right)>d_{G}\left(u_{2}, u_{i}\right)$. If $d_{H}\left(v_{1}, v_{j}\right) \geq d_{G}\left(u_{1}, u_{i}\right)$, then

$$
d_{G \otimes H}\left(\left(u_{1}, v_{1}\right),\left(u_{i}, v_{j}\right)\right)=d_{H}\left(v_{1}, v_{j}\right)<d_{H}\left(v_{1}, v_{l}\right)=d_{G \otimes H}\left(\left(u_{1}, v_{1}\right),\left(u_{k}, v_{l}\right)\right) .
$$

Now, if $d_{H}\left(v_{j}, v_{2}\right)>d_{G}\left(u_{2}, u_{i}\right)$, then $d_{H}\left(v_{l}, v_{2}\right) \geq d_{G}\left(u_{2}, u_{i}\right)=d_{G}\left(u_{2}, u_{k}\right)$ and so

$$
d_{G \bowtie H}\left(\left(u_{2}, v_{2}\right),\left(u_{i}, v_{j}\right)\right)=d_{H}\left(v_{2}, v_{j}\right) \neq d_{H}\left(v_{2}, v_{l}\right)=d_{G \otimes H}\left(\left(u_{2}, v_{2}\right),\left(u_{k}, v_{l}\right)\right) .
$$

According to the cases above, the result follows.

Theorem 18. For any connected bipartite graph $G$ and any integer $t \geq 2 D(G)+1$,

$$
\operatorname{dim}_{l}\left(P_{t} \otimes G\right)=\left\lceil\frac{t-1}{D(G)}\right\rceil+1
$$


Proof. Let $G$ and $P_{t}$ be as in the hypotheses. From $\alpha=\left\lfloor\frac{t-1}{D(G)}\right\rfloor$ and two diametral vertices $a, b \in V(G)$ we define a set $B_{\alpha}$ as follows.

If $\alpha=\frac{t-1}{D(G)}$, then

$$
B_{\alpha}=\left\{\left(u_{1}, a\right),\left(u_{D(G)+1}, b\right),\left(u_{2 D(G)+1}, a\right),\left(u_{3 D(G)+1}, b\right), \ldots,\left(u_{\alpha D(G)+1}, b\right)\right\}
$$

for $\alpha$ is odd and

$$
B_{\alpha}=\left\{\left(u_{1}, a\right),\left(u_{D(G)+1}, b\right),\left(u_{2 D(G)+1}, a\right),\left(u_{3 D(G)+1}, b\right), \ldots,\left(u_{\alpha D(G)+1}, a\right)\right\}
$$

for $\alpha$ even.

If $\alpha<\frac{t-1}{D(G)}$, then

$$
B_{\alpha}=\left\{\left(u_{1}, a\right),\left(u_{D(G)+1}, b\right),\left(u_{2 D(G)+1}, a\right),\left(u_{3 D(G)+1}, b\right), \ldots,\left(u_{\alpha D(G)+1}, b\right),\left(u_{t}, a\right)\right\}
$$

for $\alpha$ odd and

$$
B_{\alpha}=\left\{\left(u_{1}, a\right),\left(u_{D(G)+1}, b\right),\left(u_{2 D(G)+1}, a\right),\left(u_{3 D(G)+1}, b\right), \ldots,\left(u_{\alpha D(G)+1}, a\right),\left(u_{t}, b\right)\right\}
$$

for $\alpha$ even. We would point out that, in any case, $\left|B_{\alpha}\right|=\left\lceil\frac{t-1}{D(G)}\right\rceil+1$.

We will show that $B_{\alpha}$ is a local metric generator for $P_{t} \otimes G$. In order to see that, let $\left(u_{i}, v_{j}\right)$ and $\left(u_{k}, v_{l}\right)$ be two adjacent vertices belonging to $V\left(P_{t} \otimes G\right)-B_{\alpha}$. We consider, without lost of generality, that $i \leq k$ and we differentiate the following three cases for $k$.

- $1 \leq k \leq D(G)+1$. Let $T_{1}=\left\{u_{1}, \ldots, u_{D(G)+1}\right\} \times V(G)$. In this case $\left(u_{i}, v_{j}\right),\left(u_{k}, v_{l}\right) \in T_{1}$ and, by Lemma 17 the set $\left\{\left(u_{1}, a\right),\left(u_{D(G)+1}, b\right),\left(u_{2 D(G)+1}, a\right)\right\} \subset B_{\alpha}$ resolves $T_{1}$.

- $p D(G)+2 \leq k \leq(p+1) D(G)+1$, for some integer $p \in\{1, \ldots, \alpha-1\}$. Let $T_{p}=$ $\left\{u_{p D(G)+1}, \ldots, u_{(p+1) D(G)+1}\right\} \times V(G)$. In this case $\left(u_{i}, v_{j}\right),\left(u_{k}, v_{l}\right) \in T_{p}$ and we can take $x, y \in\{a, b\}$ so that $X_{p}=\left\{\left(u_{(p-1) D(G)+1}, x\right),\left(u_{p D(G)+1}, y\right),\left(u_{(p+1) D(G)+1}, x\right)\right\}$ is a subset of $B_{\alpha}$. Thus, by Lemma 17 we can conclude that $X_{p}$ resolves $T_{p}$.

- $\alpha D(G)+2 \leq k \leq t$. Let $T_{t}=\left\{u_{\alpha D(G)+1}, \ldots, u_{t}\right\} \times V(G)$. As above, $\left(u_{i}, v_{j}\right),\left(u_{k}, v_{l}\right) \in T_{t}$ and we can take $x, y \in\{a, b\}$ so that the set $X_{t}=\left\{\left(u_{(\alpha-1) D(G)+1}, x\right),\left(u_{\alpha D(G)+1}, y\right),\left(u_{t}, x\right)\right\}$ is a subset of $B_{\alpha}$. Thus, by Lemma 17 we can conclude that $X_{t}$ resolves $T_{t}$.

According to the three cases above we have $\operatorname{dim}_{l}\left(P_{t} \otimes G\right) \leq\left\lceil\frac{t-1}{D(G)}\right\rceil+1$. Therefore, by Theorem 16 we conclude the proof.

The authors of [24] conjectured that for any integers $t$ and $t^{\prime}$ such that $2 \leq t^{\prime}<t$, the metric dimension of $P_{t} \otimes P_{t^{\prime}}$ equals $\left\lceil\frac{t+t^{\prime}-2}{t^{\prime}-1}\right\rceil$. We are now able to prove the conjecture.

Theorem 19. For any integers $t$ and $t^{\prime}$ such that $2 \leq t^{\prime}<t$,

$$
\operatorname{dim}\left(P_{t} \otimes P_{t^{\prime}}\right)=\left\lceil\frac{t+t^{\prime}-2}{t^{\prime}-1}\right\rceil .
$$

Proof. As pointed out in Section 2, for $t^{\prime} \leq t \leq 2 t^{\prime}-1, \operatorname{dim}_{l}\left(P_{t} \otimes P_{t^{\prime}}\right)=3$. Now, since $\operatorname{dim}_{l}\left(P_{t} \otimes P_{t^{\prime}}\right) \leq \operatorname{dim}\left(P_{t} \otimes P_{t^{\prime}}\right)$, if $t \geq 2 t^{\prime}-1$, then by Theorem 18 we obtain the lower bound $\operatorname{dim}\left(P_{t} \otimes P_{t^{\prime}}\right) \geq\left\lceil\frac{t+t^{\prime}-2}{t^{\prime}-1}\right\rceil$. The upper bound was obtained in [24]. Therefore, the result follows. 


\section{The Particular Case of $C_{t} \otimes G$}

In this section we assume that $t$ is an integer greater than or equal to three and $V\left(C_{t}\right)=$ $\left\{u_{1}, u_{2}, \ldots, u_{t}\right\}$, where $u_{1} \sim u_{t}$ and $u_{i} \sim u_{i+1}$, for every $i \in\{1, \ldots, t-1\}$.

Lemma 20. Let $G$ be a connected graph and let $t \geq 3$ be an integer. Let $u_{i_{1}}, u_{i_{2}}, \ldots, u_{i_{b}}$ be the first components of the elements in a local metric basis of $C_{t} \otimes G$, where $i_{1} \leq i_{2} \leq \cdots \leq i_{b}$. Then for any $l \in\{1, \ldots, b\}, d_{C_{t}}\left(u_{i_{l+2}}, u_{i_{l}}\right) \leq 2 D(G)$, where the subscripts of $i$ are taken modulo $b$.

Proof. Let $B$ be a local metric basis of $C_{t} \otimes G$ and let $u_{i_{1}}, u_{i_{2}}, \ldots, u_{i_{b}}$ be the first components of the elements in $B$, where $i_{1}=1 \leq i_{2} \leq \cdots \leq i_{b}$. First of all, notice that $|B|=b$ and, by Theorem 4, $b \geq 3$.

We proceed by contradiction. Suppose that $d_{C_{t}}\left(u_{i_{l+2}}, u_{i_{l}}\right)>2 D(G)$ for some $l \in$ $\{1, \ldots, b\}$. In such a case we have that $d_{C_{t}}\left(u_{i_{l+1}}, u_{i_{l}}\right)>D(G)$ or $d_{C_{t}}\left(u_{i_{l+2}}, u_{i_{l+1}}\right)>D(G)$. We suppose that $d_{C_{t}}\left(u_{i_{l+1}}, u_{i_{l}}\right)>D(G)$, being the second case analogous. We now take $y, z \in V(G)$ such that $\left(u_{i_{l+1}}, y\right) \in B$ and $z \in N_{G}(y)$. Notice that $\left(u_{i_{l}+D(G)}, y\right)$ and $\left(u_{i_{l}+D(G)}, z\right)$ are adjacent. We differentiate the following cases for $\left(u_{i_{k}}, w\right) \in B$. If $k \neq l+1$, then $d_{C_{t}}\left(u_{i_{l}+D(G)}, u_{i_{k}}\right) \geq D(G)$ and so

$$
d_{C_{t} \bowtie G}\left(\left(u_{i_{k}}, w\right),\left(u_{i_{l}+D(G)}, y\right)\right)=d_{C_{t}}\left(u_{i_{l}+D(G)}, u_{i_{k}}\right)=d_{C_{t} \bowtie G}\left(\left(u_{i_{k}}, w\right),\left(u_{i_{l}+D(G)}, z\right)\right) .
$$

If $k=l+1$ and $i_{l+1} \neq i_{l+2}$ then $w=y$ and since $d_{C_{t}}\left(u_{i_{l+1}}, u_{i_{l}}\right)>D(G)$, we have

$$
d_{C_{t} \bowtie G}\left(\left(u_{i_{k}}, w\right),\left(u_{i_{l}+D(G)}, y\right)\right)=d_{C_{t}}\left(u_{i_{k}}, u_{i_{l}+D(G)}\right)=d_{C_{t} \bowtie G}\left(\left(u_{i_{k}}, w\right),\left(u_{i_{l}+D(G)}, z\right)\right) .
$$

If $k=l+1$ and $i_{l+1}=i_{l+2}$ then from the assumption $d_{C_{t}}\left(u_{i_{l+2}}, u_{i_{l}}\right)>2 D(G)$ we have that $d_{C_{t}}\left(u_{i_{k}}, u_{i_{l}+D(G)}\right)>D(G)$ and so

$$
d_{C_{t} \bowtie G}\left(\left(u_{i_{k}}, w\right),\left(u_{i_{l}+D(G)}, y\right)\right)=d_{C_{t}}\left(u_{i_{k}}, u_{i_{l}+D(G)}\right)=d_{C_{t} \bowtie G}\left(\left(u_{i_{k}}, w\right),\left(u_{i_{l}+D(G)}, z\right)\right) .
$$

Hence, no vertex in $B$ is able to distinguish the adjacent vertices $\left(u_{i_{l}+D(G)}, y\right)$ and $\left(u_{i_{l}+D(G)}, z\right)$, which is a contradiction. Therefore, the proof is complete.

Theorem 21. For any connected graph $G$ and any integer $t \geq 1$,

$$
\operatorname{dim}_{l}\left(C_{t} \otimes G\right) \geq\left\lceil\frac{t}{D(G)}\right\rceil .
$$

Proof. If $3 D(G) \geq t \geq 1$, then $\left[\frac{t}{D(G)}\right] \leq 3$ and, by Theorem 4, the result follows. From now on we take $t>3 D(G)$. Let $u_{i_{1}}, u_{i_{2}}, \ldots, u_{i_{b}}$ be the first components of the elements in a local metric basis $B$ of $C_{t} \otimes G$, where $i_{1}=1 \leq i_{2} \leq \cdots \leq i_{b}$. First of all, notice that $t+1-i_{b-1}=d_{C_{t}}\left(u_{i_{1}}, u_{i_{b-1}}\right)$ and so Lemma 20 leads to $i_{b-1} \geq t+1-2 D(G)$. We now differentiate two cases.

Case 1. $b$ even. In this case $b-1$ is odd and by Lemma 20 we have

$$
i_{3} \leq 2 D(G)+1, i_{5} \leq 4 D(G)+1, \ldots, i_{b-1} \leq(b-2) D(G)+1 .
$$

Hence, $t+1-2 D(G) \leq i_{b-1} \leq(b-2) D(G)+1$, so that $b \geq \frac{t}{D(G)}$. 
Case 2. $b$ odd. By Lemma 20 we have

$$
i_{3} \leq D(G)+1, i_{4} \leq 3 D(G)+1, \ldots, i_{b} \leq(b-1) D(G)+1 .
$$

Now, since $t+i_{2}-i_{b}=d_{C_{t}}\left(u_{i_{2}}, u_{b}\right) \leq 2 D(G)$, we have

$$
i_{2} \leq 2 D(G)-t+i_{b} \leq(b+1) D(G)-t+1 .
$$

Hence,

$$
i_{2} \leq(b+1) D(G)-t+1, i_{4} \leq(b+3) D(G)-t+1, \ldots, i_{b-1} \leq(2 b-2) D(G)-t+1 .
$$

Thus, $t+1-2 D(G) \leq i_{b-1} \leq(2 b-2) D(G)-t+1$, so that $b \geq \frac{t}{D(G)}$.

Theorem 22. For any connected bipartite graph $G$ and any integer $t \geq 4 D(G)$,

$$
\operatorname{dim}_{l}\left(C_{t} \otimes G\right) \leq\left\lceil\frac{t}{D(G)}\right\rceil+1 .
$$

Furthermore, if $\left\lceil\frac{t}{D(G)}\right\rceil$ is even, then

$$
\operatorname{dim}_{l}\left(C_{t} \otimes G\right)=\left\lceil\frac{t}{D(G)}\right\rceil .
$$

Proof. Let $G$ and $C_{t}$ be as in the hypotheses. From $\alpha=\left\lceil\frac{t}{D(G)}\right\rceil$ and two diametral vertices $a, b \in V(G)$ we define a set $B_{\alpha}$ as follows. If $\alpha$ is even, then

$$
B_{\alpha}=\left\{\left(u_{1}, a\right),\left(u_{D(G)+1}, b\right),\left(u_{2 D(G)+1}, a\right),\left(u_{3 D(G)+1}, b\right), \ldots,\left(u_{(\alpha-1) D(G)+1}, b\right)\right\}
$$

and, if $\alpha$ is odd, then

$$
B_{\alpha}=\left\{\left(u_{1}, a\right),\left(u_{D(G)+1}, b\right),\left(u_{2 D(G)+1}, a\right),\left(u_{3 D(G)+1}, b\right), \ldots,\left(u_{(\alpha-1) D(G)+1}, a\right),\left(u_{(\alpha-1) D(G)+1}, b\right)\right\} .
$$

Notice that $\left|B_{\alpha}\right|=\alpha$, for $\alpha$ even, and $\left|B_{\alpha}\right|=\alpha+1$, for $\alpha$ odd. We will show that $B_{\alpha}$ is a local metric generator for $C_{t} \otimes G$. In order to see that, let $\left(u_{i}, v_{j}\right),\left(u_{k}, v_{l}\right)$ be a pair of adjacent vertices belonging to $V\left(C_{t} \otimes G\right)-B_{\alpha}$. We consider, without lost of generality, that $i \leq k$ and we differentiate the following three cases for $k$.

- $2 \leq k \leq D(G)+1$. Let $T_{1}=\left\{u_{1}, \ldots, u_{D(G)+1}\right\} \times V(G)$. In this case $\left(u_{i}, v_{j}\right),\left(u_{k}, v_{l}\right) \in T_{1}$ and, by Lemma 17 the set $\left\{\left(u_{1}, a\right),\left(u_{D(G)+1}, b\right),\left(u_{2 D(G)+1}, a\right)\right\} \subset B_{\alpha}$ resolves $T_{1}$.

- $p D(G)+2 \leq k \leq(p+1) D(G)+1$, for some integer $p \in\{1, \ldots, \alpha-2\}$. Let $T_{p}=$ $\left\{u_{p D(G)+1}, \ldots, u_{(p+1) D(G)+1}\right\} \times V(G)$. In this case $\left(u_{i}, v_{j}\right),\left(u_{k}, v_{l}\right) \in T_{p}$ and we can take $x, y \in\{a, b\}$ such that $X_{p}=\left\{\left(u_{(p-1) D(G)+1}, x\right),\left(u_{p D(G)+1}, y\right),\left(u_{(p+1) D(G)+1}, x\right)\right\}$ is a subset of $B_{\alpha}$. Thus, by Lemma 17 we can conclude that $X_{p}$ resolves $T_{p}$.

- $(\alpha-1) D(G)+2 \leq k \leq t+1$. Let $T_{t}=\left\{u_{(\alpha-1) D(G)+1}, \ldots, u_{t+1}\right\} \times V(G)$. In this case, $\left(u_{i}, v_{j}\right),\left(u_{k}, v_{l}\right) \in T_{t}$ and we take the set $X_{t}=\left\{\left(u_{(\alpha-1) D(G)+1}, b\right),\left(u_{1}, a\right),\left(u_{D(G)+1}, b\right)\right\} \subset$ $B_{\alpha}$. By Lemma 17 we can conclude that $X_{t}$ resolves $T_{t}$.

According to the three cases above $B_{\alpha}$ is a local metric generator for $C_{t} \otimes G$ and so $\operatorname{dim}_{l}\left(C_{t} \otimes G\right) \leq\left|B_{\alpha}\right|$. Therefore, by Theorem 21 we conclude the proof. 


\section{References}

[1] R. F. Bailey, K. Meagher, On the metric dimension of grassmann graphs, Discrete Mathematics \& Theoretical Computer Science 13 (4) (2011) 97-104.

URL http://www .dmtcs .org/dmtcs-ojs/index.php/dmtcs/article/view/2049

[2] G. Barragán-Ramírez, C. G. Gómez, J. A. Rodríguez-Velázquez, Closed formulae for the local metric dimension of corona product graphs, Electronic Notes in Discrete Mathematics 46 (0) (2014) 27-34.

URL http://www.sciencedirect.com/science/article/pii/S1571065314000067

[3] L. M. Blumenthal, Theory and applications of distance geometry, Second edition, Chelsea Publishing Co., New York, 1970.

[4] R. C. Brigham, G. Chartrand, R. D. Dutton, P. Zhang, Resolving domination in graphs, Mathematica Bohemica 128 (1) (2003) 25-36.

URL http://mb.math.cas.cz/mb128-1/3.html

[5] J. Cáceres, C. Hernando, M. Mora, I. M. Pelayo, M. L. Puertas, C. Seara, D. R. Wood, On the metric dimension of cartesian product of graphs, SIAM Journal on Discrete Mathematics 21 (2) (2007) 423-441.

URL http://epubs .siam.org/doi/abs/10.1137/050641867

[6] G. Chartrand, L. Eroh, M. A. Johnson, O. R. Oellermann, Resolvability in graphs and the metric dimension of a graph, Discrete Applied Mathematics 105 (1-3) (2000) 99113.

URL http://dx.doi .org/10.1016/S0166-218X(00)00198-0

[7] G. Chartrand, V. Saenpholphat, P. Zhang, The independent resolving number of a graph, Mathematica Bohemica 128 (4) (2003) 379-393.

URL http://mb.math.cas.cz/mb128-4/4.html

[8] A. Estrada-Moreno, J. A. Rodríguez-Velázquez, I. G. Yero, The $k$-metric dimension of a graph, Applied Mathematics \& Information Sciences. To appear.

URL http://arxiv.org/abs/1312.6840

[9] M. Feng, K. Wang, On the metric dimension of bilinear forms graphs, Discrete Mathematics 312 (6) (2012) 1266 - 1268.

URL http://www.sciencedirect.com/science/article/pii/S0012365X11005279

[10] H. Fernau, J. A. Rodríguez-Velázquez, On the (adjacency) metric dimension of corona and strong product graphs and their local variants: combinatorial and computational results, arXiv:1309.2275 [math.CO].

URL http://arxiv-web3. library.cornell .edu/abs/1309.2275

[11] H. Fernau, J. A. Rodríguez-Velázquez, Notions of metric dimension of corona products: combinatorial and computational results, in: Computer science - theory and applications, vol. 8476 of Lecture Notes in Comput. Sci., Springer, Cham, 2014, pp. 153-166.

[12] J. Guo, K. Wang, F. Li, Metric dimension of some distance-regular graphs, Journal of Combinatorial Optimization 26 (2013) 190-197.

URL http://dx .doi .org/10.1007/s10878-012-9459-x 
[13] R. Hammack, W. Imrich, S. Klavžar, Handbook of product graphs, Discrete Mathematics and its Applications, 2nd ed., CRC Press, 2011.

URL http://www . crcpress . com/product/isbn/9781439813041

[14] F. Harary, R. A. Melter, On the metric dimension of a graph, Ars Combinatoria 2 (1976) 191-195.

URL http://www .ams .org/mathscinet-getitem?mr=0457289

[15] T. W. Haynes, M. A. Henning, J. Howard, Locating and total dominating sets in trees, Discrete Applied Mathematics 154 (8) (2006) 1293-1300.

URL http://www.sciencedirect.com/science/article/pii/S0166218X06000035

[16] M. Johnson, Structure-activity maps for visualizing the graph variables arising in drug design, Journal of Biopharmaceutical Statistics 3 (2) (1993) 203-236, pMID: 8220404. URL http://www .tandfonline.com/doi/abs/10.1080/10543409308835060

[17] M. Johnson, Browsable structure-activity datasets, in: R. Carbó-Dorca, P. Mezey (eds.), Advances in Molecular Similarity, chap. 8, JAI Press Inc, Stamford, Connecticut, 1998, pp. $153-170$.

URL http://books .google.es/books?id=1vvMsHXd2AsC

[18] S. Khuller, B. Raghavachari, A. Rosenfeld, Landmarks in graphs, Discrete Applied Mathematics 70 (3) (1996) 217-229.

URL http://www.sciencedirect.com/science/article/pii/0166218X95001062

[19] R. A. Melter, I. Tomescu, Metric bases in digital geometry, Computer Vision, Graphics, and Image Processing 25 (1) (1984) 113-121.

URL http://www.sciencedirect.com/science/article/pii/0734189X84900513

[20] F. Okamoto, B. Phinezy, P. Zhang, The local metric dimension of a graph, Mathematica Bohemica 135 (3) (2010) 239-255.

URL http://dml.cz/dmlcz/140702

[21] Y. Ramírez-Cruz, O. R. Oellermann, J. A. Rodríguez-Velázquez, Simultaneous resolvability in graph families, Electronic Notes in Discrete Mathematics 46 (0) (2014) 241 248.

URL http://www.sciencedirect.com/science/article/pii/S157106531400033X

[22] J. A. Rodríguez-Velázquez, G. A. Barragán-Ramírez, C. García Gómez, On the local metric dimension of corona product graphs, Bulletin of the Malaysian Mathematical Sciences Society. (2015) To appear.

URL http://arxiv-web3.library.cornell.edu/abs/1308.6689

[23] J. A. Rodríguez-Velázquez, C. García Gómez, G. A. Barragán-Ramírez, Computing the local metric dimension of a graph from the local metric dimension of primary subgraphs, Int. J. Comput. Math. 92 (4) (2015) 686-693.

URL http://arxiv.org/abs/1402.0177

[24] J. A. Rodríguez-Velázquez, D. Kuziak, I. G. Yero, J. M. Sigarreta, The metric dimension of strong product graphs., Carpathian Journal of Mathematics 31 (2) (2015) 261-268. 
[25] V. Saenpholphat, P. Zhang, Conditional resolvability in graphs: a survey, International Journal of Mathematics and Mathematical Sciences 2004 (38) (2004) 1997-2017.

URL http://www .hindawi .com/journals/ijmms/2004/247096/abs/

[26] A. Sebö, E. Tannier, On metric generators of graphs, Mathematics of Operations Research 29 (2) (2004) 383-393.

URL http://dx.doi.org/10.1287/moor.1030.0070

[27] P. J. Slater, Leaves of trees, Congressus Numerantium 14 (1975) 549-559.

[28] I. G. Yero, D. Kuziak, J. A. Rodríquez-Velázquez, On the metric dimension of corona product graphs, Computers \& Mathematics with Applications 61 (9) (2011) 2793-2798. URL http://www.sciencedirect.com/science/article/pii/S0898122111002094 